\title{
Preparación de un nuevo material híbrido orgánico/inorgánico mediante la intercalación de colina en bronce de molibdeno
}

\author{
P. BENITO', F.M. LABAJOS ${ }^{1,2}$, P. MORENO ${ }^{2}$, W. JONES ${ }^{3}$ \\ 1Departamento de Química Inorgánica. Universidad de Salamanca E.T.S. Ingeniería Industrial, Béjar 37700 Salamanca. \\ ${ }^{2}$ Departamento de Ingeniería Mecánica. Universidad de Salamanca E.T.S. Ingeniería Industrial, Béjar 37700 Salamanca. \\ ${ }^{3}$ Departamento of Chemistry. University of Cambridge. Lensfield Road, CB2 1EW. Cambridge, U.K.
}

\begin{abstract}
Se aborda la intercalación de un compuesto orgánico, 2-hidroxil etil trimetil amonio (Colina), entre las láminas de bronce azul de molibdeno. El sólido obtenido se caracteriza mediante análisis químico (AQ), difracción de rayos-X (XRD), análisis térmico (DTA y TG) y espectroscopia infrarroja (FT-IR). La utilización de un bronce sódico de molibdeno recién preparado facilita la incorporación de la molécula orgánica, dando lugar a la formación de una única fase laminar. El material híbrido muestra un alto ordenamiento laminar, estable a temperaturas inferiores a los $550 \mathrm{~K}$, que se descompone por combustión del componente orgánico. Los resultado obtenidos indican que el bronce azul de molibdeno puede ser un buen candidato para la obtención de compuestos de intercalación.
\end{abstract}

Palabras clave: Bronce de Molibdeno, Hibrido orgánico-inorgánico, intercalación.

Preparation of a new hybrid organic/inorganic material by cholina intercalation into molybdenum bronzes

Intercalation of a organic compound, 2-hydroxyl etil trimetil ammonium, in the layered host molybdenum bronzes was achieved. Full characterization of solids was performed using chemical analysis, X-ray diffaction, thermal analysis and IR spectroscopy. Using fresh sodium hydrated layered molybdenum bronce yields a monofasic layered solid. The hybrid organic-inorganic compound exhibs a high lamellar order, which is stable at temperatura below $550 \mathrm{~K}$, then collapsing by combustion of organic component. All features show that blue molybdenum bronzes will be a good host for intercalation.

Key words: Molybdenum bronze, hybrid organic-inorganic, intercalation

\section{INTRODUCCIÓN}

La búsqueda de nuevos materiales compuestos para mejorar propiedades tales como microporosidad, actividad catalítica, propiedades electrónicas, propiedades mecánicas, etc., supone una activa línea de trabajo en la química del estado sólido (1). En las últimas décadas se han logrado grandes avances en el desarrollo de materiales tecnológicos con alto valor añadido basados en compuestos con estructura abierta 3D, formados a partir de estructuras laminares 2D (arcillas catiónicas, hidróxidos dobles laminares, sulfuros y óxidos de metales de transición, etc.). Estos materiales pueden albergar moléculas orgánicas entre sus láminas, dando lugar a híbridos orgánicos-inorgánicos, que han despertado el interés por la formación de nuevos compuestos sólidos muy interesantes: materiales con propiedades conductoras, magnéticas y ópticas (2-5), o los más recientemente empleados por la mejora de sus propiedades mecánicas e ignífugas (6).

El trióxido de molibdeno es un ejemplo de material con estructura laminar que puede experimentar reacciones de intercalación. La estructura del $\mathrm{MoO}_{3}$ puede ser descrita como infinitas cadenas de octaedros $\mathrm{MoO}_{6}$ que comparten vértices y ejes dando lugar a la formación de láminas onduladas, de espesor $6.928 \AA$, que se colocan paralelas entre sí, pudiéndose ser representadas por la fórmula MoO1/1O2/2O3/3 (que indica que el Mo está octaédricamente coordinado a un átomo de oxígeno no compartido, a dos átomos de oxígeno compartidos con otro átomo de molibdeno y a tres átomos de oxígeno compartidos con otros dos átomos de molibdeno) $(7,8)$. En este trabajo se describe la inclusión de una sustancia orgánica en bronce sódico de molibdeno, previamente sintetizado. Este proceso supone la primera etapa para la incorporación de monómeros en sistemas inorgánicos que sirvan de precursores para la obtención de un polímero orgánico en matriz inorgánica.

\section{EXPERIMENTAL}

Se prepararon muestras de bronce azul de molibdeno siguiendo el método descrito por Thomas y McCarron (10), que supone una excelente vía para la introducción de cationes alcalinos hidratados entre las láminas $\mathrm{MoO}_{3}$. El proceso, de modo simplificado, consiste en la adición de $\mathrm{Na}_{2} \mathrm{MoO}_{4}$ y $\mathrm{Na}_{2} \mathrm{~S}_{2} \mathrm{O}_{4}$ sobre una suspensión de $\mathrm{MoO}_{3^{\prime}}$ manteniendo en todo momento burbujeo de nitrógeno. El sólido azul que se forma se recogió por filtración y fue lavado con agua bidestilada hasta ausencia total de color en el filtrado. Para la caracterización de los distintos sólido se emplearon difractómetros Siemens D500 y Philips PW 1710/00 para registrar los difractogramas de rayos X. Los análisis químicos fueron realizados mediante fluorescencia de rayos- $X$ en un espectrómetro Philips PW 1400. Los espectros infrarrojos se registraron en un Nicolet 205 FT-IR MX. Por lo que respecta a los análisis térmicos, se emplearon los equipos Perkin-Elmer DTA7 y TG7.

Los datos obtenidos de los análisis químico y térmico para el bronce sódico son: $9.8 \%$ de sodio, $58.4 \%$ de molibdeno y $5.3 \%$ de agua. La caracterización mediante difracción de rayos $\mathrm{X}$, confirma que la única especie cristalina es el bronce azul de molibdeno, $\left[\mathrm{Na}\left(\mathrm{H}_{2} \mathrm{O}\right)_{n}\right]_{x} \mathrm{MoO}_{3}$. El sólido obtenido presenta un alto grado de cristalinidad y un buen orden laminar, dando lugar a picos agudos y con una excelente concordancia entre los valores de los picos originados por planos (0k0). Por lo que respecta a su comportamiento frente a la temperatura, el sólido presenta un efecto endotérmico, debido a la deshidratación del catión interlaminar, como se desprende en el registro de análisis térmico diferencial, Figura 1. Los valores de pérdida de peso debido a esa etapa de deshidratación son consistente con la fórmula química $\left[\mathrm{Na}\left(\mathrm{H}_{2} \mathrm{O}\right)_{2}\right]_{0.273} \mathrm{MoO}_{3}$. La presencia en DTA de un pico exotérmico agudo, próximo a los $300{ }^{\circ} \mathrm{C}$, es característica de estructuras de bronces 


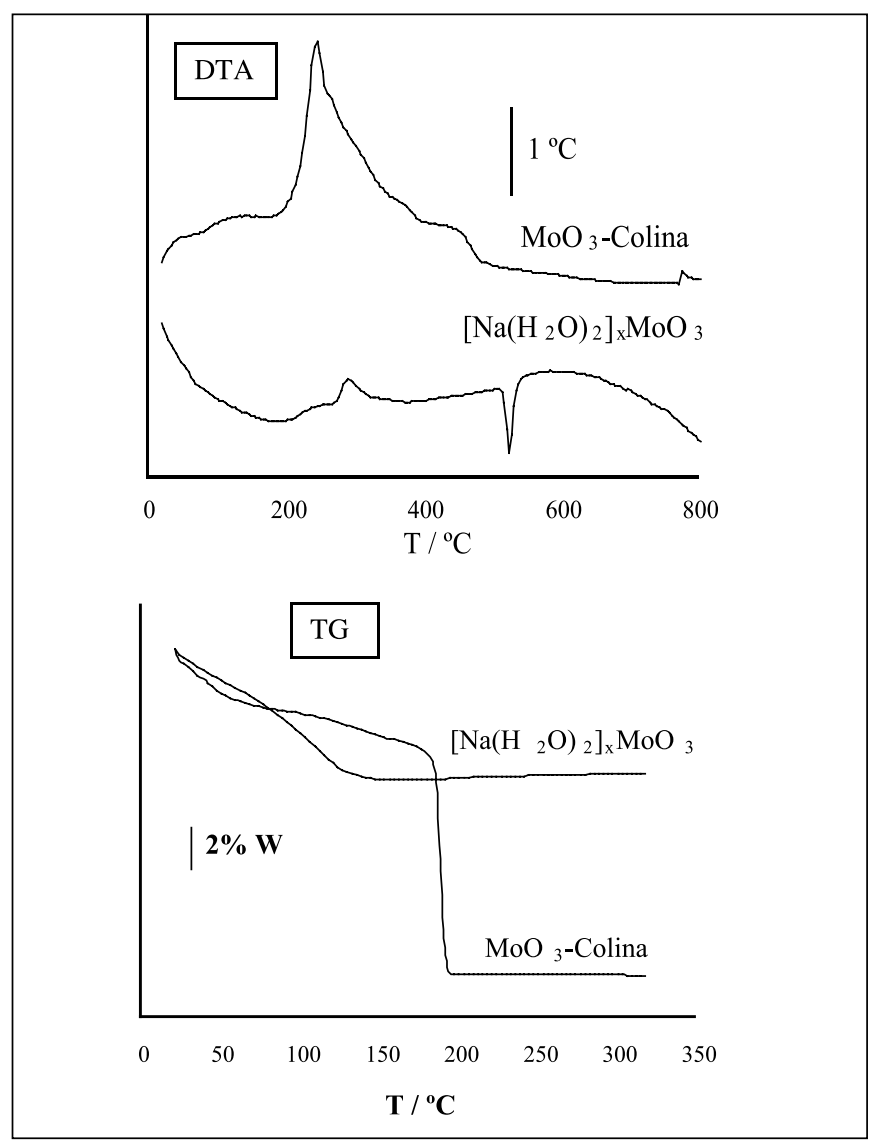

Figura 1.- Curvas de análisis térmico (DTA y TG) de las muestras que se indican.

hidratados, estando asociado con una transformación de fase desde el bronce azul de molibdeno (blue bronze) hacia la formación de bronce sódico de molibdeno púrpura (purple bronze) (11). Estos materiales experimentan fácilmente procesos de "hinchazón-deshinchazón" como lo pone de manifiesto la variación del espaciado correspondiente a la zona interlaminar cuando se registra el difractograma del óxido húmedo $\left(\mathrm{d}_{(020)}=11.39 \AA\right.$ ) y seco $\left(\mathrm{d}_{(020)}=9.66 \AA\right)$. Estos resultados son acordes con los encontrados en bibliografía (12), y se basa en variaciones estructurales. Para la muestra seca, se propone una estructura donde el ion sodio está coordinado a dos moléculas de agua y cuatro oxígenos terminales $(\mathrm{Mo}=\mathrm{O})$ del óxido de molibdeno laminar. Por lo que respecta a la muestra hidratada, el sodio está coordinado octaédricamente a seis moléculas de agua, que se sitúan entre los cationes sodio y los átomos de oxígeno del enlace $\mathrm{Mo}=\mathrm{O}$ laminar.

Este sólido, bronce azul de molibdeno, se utilizó como precursor para la inclusión del compuesto orgánico por cambio iónico. El procedimiento seguido consistió en la adición de 2-hidroxil etil trimetil amonio (Colina) a una suspensión que contenía de $2 \mathrm{~g}$ de bronce azul de molibdeno recién preparado, en proporción 3:1. El sólido resultante se recogió por filtración y el filtrado se lavó con etanol. La utilización de bronce recién preparado, sin secar, se debe a la ventaja que supone disponer de un mayor espaciado interlaminar(4,45 ̊̊ frente a $2.60 \AA$ ), lo que facilita el acceso a la región interlaminar, además de evitar una conversión, no deseada, del bronce sódico hacia formas protonadas, que son más inertes al cambio catiónico. Por su parte, el estudio mediante análisis químico proporciona los contenidos porcentuales que se indican: $59.50 \%$ de Mo, $6.12 \%$ de C, $1.30 \%$ de $\mathrm{H}$ y $1.28 \%$ de $\mathrm{N}$. Estos resultados conducen a establecer la fórmula del compuesto

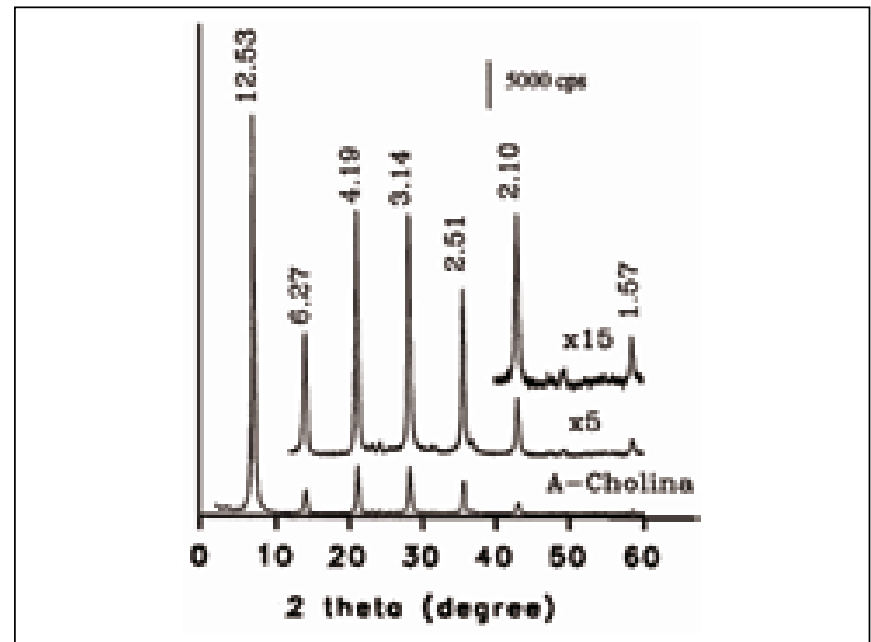

Figura 2.- Diagrama de difracción de rayos $\mathrm{X}$ del compuesto A-Colina $\left(\mathrm{A}=\left[\mathrm{Na}\left(\mathrm{H}_{2} \mathrm{O}\right)_{2}\right]_{x} \mathrm{MoO}_{3}\right)$

como $\left(\mathrm{C}_{5} \mathrm{H}_{14} \mathrm{NO}\right)_{0165} \mathrm{MoO}_{3}$. La cantidad de compuesto orgánico es ligeramente menor de la cantidad teórica esperada, lo que se debe, sin duda, a una parcial reducción del óxido de molibdeno durante la etapa de cambio iónico, favorecida por el carácter básico del compuesto orgánico. En la Figura 2 se muestra el difractograma de rayos $\mathrm{X}$ de la muestra A-Colina recién obtenida $\left(\mathrm{A}=\left[\mathrm{Na}\left(\mathrm{H}_{2} \mathrm{O}\right)_{2}\right]_{x} \mathrm{MoO}_{3}\right)$. El registro se corresponde con el de un material laminar altamente cristalino. La buena resolución del difractograma permite detectar hasta siete reflexiones originadas por planos (0k0) apilados a lo largo del eje b. La buena correlación que existe entre el valor $\mathrm{d}_{(020)}=12.53 \AA$, y el resto de los valores $\mathrm{d}(0 \mathrm{k} 0)$ indica que el producto presenta una estructura laminar altamente ordenada. Por otra parte, la secuencia de intensidades relativas de los picos registrados es característica de compuestos laminares de $\mathrm{MoO}_{3}$ tipo sandwich, que debido a la incorporación de moléculas de distinto tamaño en su región interlaminar producen un aumento del espaciado basal. La distancia entre las láminas de molibdeno en el caso de la muestra A-Colina es de $5.61 \AA ̊$, que se corresponde con una ocupación simple de una molécula de Colina que se ubica perpendicular a las láminas a lo largo del eje b (13).

Los datos registrados en la realización del estudio del material híbrido mediante espectroscopía infrarroja indican que se trata del material compuesto. Los registros del sólido orgánico/inorgánico muestran bandas correspondientes a todos los grupos moleculares presentes en el mismo. Además de las bandas a 582 y $820 \mathrm{~cm}^{-1}$, originadas por la vibración de tensión Mo-O, se registran bandas debidas a los enlaces $\mathrm{C}-\mathrm{H}$, $\mathrm{C}-\mathrm{O}$ y $\mathrm{O}-\mathrm{H}$ a valores de número de ondas consistentes con los dados en literatura para las sales de amonio (14). Los intensos picos registrados a 3410 y $1640 \mathrm{~cm}^{-1}$ son consecuencia de la presencia de grupos hidroxilo en el catión orgánico. Muy próximos al pico originado por la vibración de tensión O-H se registran picos agudos de baja intensidad con mínimos a 2965, 2930 y $2854 \mathrm{~cm}^{-1}$; la asignación de estos picos en sólidos laminares con grupos hidroxilo suele atribuirse a la formación de enlaces de hidrógeno (15). Teniendo en cuenta la composición del compuesto $\mathrm{MoO}_{3}$-Colina, no parece muy adecuado aceptar que haya formación de enlaces de hidrógeno. Por otra parte, esta región del espectro es una de las zonas más activas en infrarrojo para compuestos orgánicos, lo que hace pensar que las dos bandas mencionadas se deben a los grupos metilo del catión orgánico. Esta suposición se ve confirmada con la presencia de dos bandas originadas por grupos C-H, una a $1475 \mathrm{~cm}^{-1}$, debida a la vibración de deformación del enlace $\mathrm{C}-\mathrm{H}$, y un hombro a $730 \mathrm{~cm}^{-1}$ que se adscribe a un movimiento "rocking" (deformación angular fuera del plano) del grupo C-H. La intensidad de esta banda está 
directamente relacionada con el número de metilenos presentes en el compuesto. Por tanto, la banda a $2965 \mathrm{~cm}^{-1}$ se debe a la vibración de tensión asimétrica del grupo metilo $\left(\mathrm{CH}_{3}\right)$, la banda registrada a $2930 \mathrm{~cm}^{-1}$ se asigna a la vibración de tensión asimétrica del grupo metileno $\left(\mathrm{CH}_{2}\right)$ y por lo que respecta a la banda a $2854 \mathrm{~cm}^{-1}$ se debe a la vibración de tensión simétrica del grupo metileno. En cuanto a la banda de tensión simétrica del grupo metilo, que debería aparecer sobre los $2875 \mathrm{~cm}^{-1}$, no se puede afirmar su presencia aunque puede intuirse un hombro sobre el pico originado por el modo equivalente del grupo metileno. A 1180 $\mathrm{cm}^{-1}$ se aprecia un mínimo poco agudo en una banda ancha que se extiende desde los $1200 \mathrm{~cm}^{-1}$ a los $1000 \mathrm{~cm}^{-1}$. En esta región se registran las vibraciones de tensión C-O y C-N de alcoholes primarios y de enlaces primarios carbono-nitrógeno. El pico intenso a $995 \mathrm{~cm}^{-1}$, con un hombro a $1000 \mathrm{~cm}^{-1}$, es el más indicativo de la existencia de óxido de molibdeno y colina; sobre los $1000 \mathrm{~cm}^{-1}$ aparecen las bandas originadas por la presencia de $\mathrm{MoO}_{3}(16)$, pero también son características las bandas de aminas cuaternarias a valores próximos a los $1000 \mathrm{~cm}^{-1}$. La evolución de esta banda para los espectros de los productos de calcinación del híbrido $\mathrm{MoO}_{3}$-Colina presenta un desdoblamiento para la muestra calcinada a $573 \mathrm{~K}$ (1011 y $\left.976 \mathrm{~cm}^{-1}\right)$ y un único pico más agudo para el producto de calcinación a $773 \mathrm{~K}$, valor al cual ya se ha eliminado el compuesto orgánico por combustión. Este comportamiento confirma que la anchura de dicha banda se debe al solapamiento de los modos de vibración originados por el óxido de molibdeno, $1000 \mathrm{~cm}^{-1}$, y por la sal de amonio cuaternaria de la molécula orgánica intercalada, $960 \mathrm{~cm}^{-1}$. Las bandas a 998, 850 y $596 \mathrm{~cm}^{-1}$ muestran un aumento en su intensidad con la temperatura de calcinación, debido a la formación de trióxido de molibdeno por la eliminación de la colina.

La evolución, con la temperatura, del sólido laminar intercalado se analizó mediante difracción de rayos $\mathrm{X}$ de los productos de calcinación obtenidos a las temperaturas de 338, 373, 453, 573 y $773 \mathrm{~K}$. Las registros ponen de manifiesto que la estructura laminar se mantiene para temperaturas inferiores a los $573 \mathrm{~K}$, valor al que se registraba la pérdida de peso principal en el estudio por análisis térmico (figura 1). Para temperaturas de calcinación superiores a los $773 \mathrm{~K}$, sólo se detecta óxido de molibdeno, debido a la total eliminación del compuesto orgánico por combustión. La curva termogravimétrica indica que a esa temperatura ya se han eliminado la práctica totalidad de los volátiles del compuesto.

A fin de completar el conocimiento de la bondad del bronce sódico de molibdeno como intermedio en procesos de intercalación, se realizaron intercalaciones utilizando cationes tipo Keggin $\left[\mathrm{ALO}_{4}(\mathrm{OH})_{24}\left(\mathrm{H}_{2} \mathrm{O}\right)_{12}\right]$ y $\left[\mathrm{Zr}_{4}(\mathrm{OH})_{8}\left(\mathrm{H}_{2} \mathrm{O}\right)_{16}\right]$, bien conocidos por su buen comportamiento en el pilareado de arcillas. Los resultados obtenidos se aprecian en la Figura 3, donde se recogen los difractogramas de rayos $\mathrm{X}$ de los sólidos resultantes recién preparados. La buena resolución, la nitidez de los picos y su gran intensidad, hacen pensar que el $\left[\mathrm{Na}\left(\mathrm{H}_{2} \mathrm{O}\right)_{2}\right]_{x}\left[\mathrm{MoO}_{3}\right]$ puede ser un buen material para albergar entre sus láminas cationes voluminosos. A la vista de la intensidad de los picos (002) y (004), los dos primeros registrados, se puede afirmar que la formación del compuesto laminar está favorecido por el carácter nucleófilo de la molécula intercalante.

\section{CONCLUSIONES}

Se ha obtenido un compuesto híbrido orgánico inorgánico, de alta homogeneidad cristalina, por desplazamiento de cationes alcalinos hidratados en la estructura de bronce azul de molibdeno. Este compuesto supone ampliar el conocimiento al estudio de intercalaciones en estructuras laminares de $\mathrm{MoO}_{3}$. Finalmente, los datos obtenidos en la inclusión de policationes en la estructura de bronce de molibdeno, confirman que el carácter nucleófilo de la molécula que se alberga entre las láminas es un factor determinante en el proceso de intercalación.

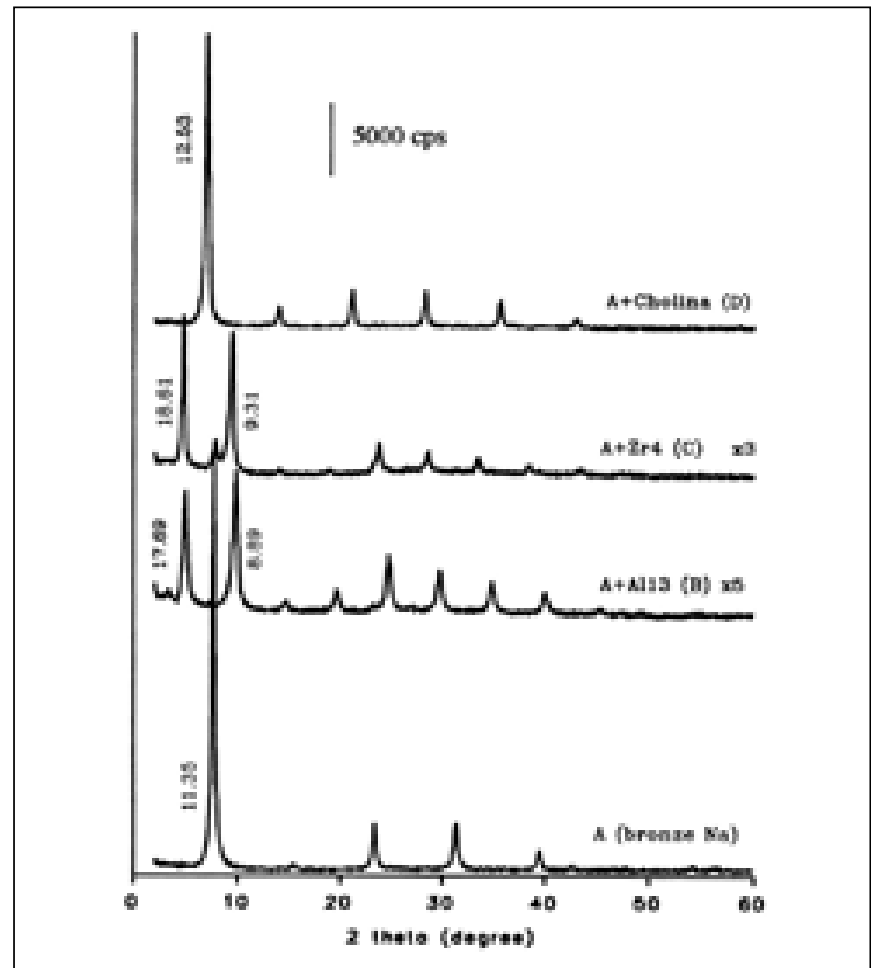

Figura 3.- Diagramas de difracción de rayos $\mathrm{X}$ de los compuestos laminares obtenidos por intercalación de $\mathrm{MoO}_{3}$ con las sustancias que se indican.

\section{AGRADECIMIENTOS}

Los autores desean agradecer la financiación recibida de la JCyL y de la Fundación "Memoria Samuel Solorzano Barruso" de la USAL (YARY).

\section{BIBLIOGRAFÍA}

1. Mark, J.E., Lee, C. Y-C and Bianconi, P.A. (Editors), Hybrid organic-inorganic composites. ACS, Washington, DC 1995.

2. Karrado, K.A., Syntthetic organo-and polymer-clays: preparation, characterization, and materials applications. Appl. Clay Sci., 17 (2000) 1-23.

3. Ardel, G., Golodnitsky, D., Feedman, K., Peled, E., Appetecchi, G.B., Romagnoli, P., Scrosati, B., Rechargable lithium/hybrid-electrolyte/pyrite battery, Journal of Powder Sources, 110 (2002) 152-162.

4. Wortham, E., Zorko, A., Arcon, D., Lappas, A., Organic-inorganic perovskites for magnetic nanocomposites, Physica B, 318 (2002) 387-391.

5. Cho, J. W., Sul, K., Characterization and properties of hybrid composites preparated from poly(vinlydene fluoride-tetrafluorethylene) and $\mathrm{SiO}_{2}$, Polymer, 42 (2002) 727-736.

6. Park, C., Park, O., Gon Lim, J., Joon Kim, H., The fabrication of cyndiotactic polystyrene/organophilic clay nanocomposites and their properties, Polymer, 41 (2001) 7465-7475.

7. Andersson G. y Magneli, A. On the Crystal Structure of Molybdenum Trioxide. Acta Chemia Scandinavica, 4 (1950) 793-797.

8. Hulliger, F. "Structural Chemistry of Layer-Type Phases" Reidel, D., Boston, 1976,

169 y ss.
9. Thomas D.M., McCarron, E.M. III, The composition and propose structure of the alkali metal layered moybdenum bronzes, Mat. Res. Bull., 21 (1986) 945-960.

10. Eda, K. Hatayama, F., Kunitomo, M., Kohmoto, T., Sotani, N., Preparation and Charaterization of a Sodium Insertion Compound of Hydrogen Molygdenum Bronze $\left[\mathrm{Na}_{0.25}\left(\mathrm{H}_{2} \mathrm{O}\right)_{\mathrm{y}}\right]\left[\mathrm{H}_{0.21} \mathrm{MoO}_{3}\right]$, J. Mater Chem., 4(2) (1994) 205-207.

11. Nazar, L.F., Wu, H., Power, W.P., J. Mater. Chem., 5 (1995) 1985-1993.

12. Kerr, T.A., Wu, H. Nazar, L.F., Concurrent Polymeriztion and Insertion of Aniline in Molybdenum Trioxide. Formation and Properties of a [Poly(aniline) $]_{0.24} \mathrm{MoO}_{3}$ Nanocompositem, Chem. Mater. 8 (1996) 2005-2015.

13. Hjortås, J., Sørum, H., A Re-investigación of the Cristal Structure of Choline Chloride, Acta Cryst., B27 (1971) 1320-1323.

14. Colthup, N.B., Daly, L.H., Wiberley, S.E., Introduction to Infrarred and Raman Spectroscopy, $3^{\mathrm{a}}$ Edición. Academic Press. San Diego, California, 1990.

15. Labajos, F.M., Rives, V., Ulibarri, M.A., A FT-IR and V-UV Spectroscopic Study of Nickel-Containing Hydrotalcite-like Compounds, [Ni(1-x)Alx (OH)2](CO3)x/2 of Nickel-Containing Hydrotalcite-like Com
nH2O, Spectroscopy Lett., 24 (1991) 499-508.

16. Mortimer, R. Powell, J.G., Greenblatt, M., McCarrol, W.H., Ramanujachary. K.V., Variable-temperature Infrared Spectroscopic Sstudy of some Moybdenum Bronzes: Evidence for Electron-Phonon coupling, J. Chem. Soc. Faraday Trans. 89(18) (1993) 3603-3609. 